\title{
International Microsurgery Club: An Effective Online Collaboration System
}

\author{
Evelyn Ting-Hsuan Tang, MD ${ }^{1,2}$ Mei Goh, MD ${ }^{3}$ Raymond Goh, MBBS, FRACS ${ }^{4}$ \\ Samir M. Ghoraba, MD ${ }^{5}$ Mark Shafarenko, MD ${ }^{6}$ Pei-Tzu Hung, MD ${ }^{1,7,8}$ Jung-Ju Huang, MD ${ }^{1}$ \\ Cheng-Fong Chu, MD ${ }^{1}$ Soo-Ha Kwon, MD ${ }^{1}$ Angela Ting-Wei Hsu, MD ${ }^{1,2}$ Jennifer An-Jou Lin, MD ${ }^{1}$ \\ Che-Hsiung Lee, $\mathrm{MD}^{1}$ Tommy Nai-Jen Chang, $\mathrm{MD}^{1}$ \\ ${ }^{1}$ Department of Plastic and Reconstructive Surgery, Linkou Medical \\ Address for correspondence Tommy Nai-Jen Chang, MD, \\ Center, Chang Gung Memorial Hospital, Chang Gung Medical \\ College, Chang Gung University, Taoyuan, Taiwan \\ 2 Department of Medicine, Mackay Medical College, New Taipei City, \\ Taiwan \\ Division of Reconstructive Microsurgery, Department of Plastic \\ and Reconstructive Surgery, Chang Gung Memorial Hospital, \\ No. 5, Fu-Hsing St. Kwei-Shan, Taoyuan 333, Taiwan \\ ${ }^{3}$ Department of Plastic and Reconstructive surgery, Gold Coast \\ (e-mail: tommynjchang@yahoo.com.tw). \\ University Hospital, Queensland, Australia \\ ${ }^{4}$ Valley Plastic Surgery, Queensland, Australia \\ ${ }^{5}$ Department of Plastic and Reconstructive Surgery, Faculty of \\ Medicine, Tanta University, Tanta, Egypt \\ 6 Faculty of Medicine, University of Toronto, Toronto, Ontario, Canada \\ ${ }^{7}$ Department of Medicine, Taipei Medical University, Taipei, Taiwan \\ 8 Shuang Ho Hospital, Ministry of Health and Welfare, Taipei Medical \\ University, New Taipei City, Taiwan \\ J Reconstr Microsurg 2020;36:412-419.
}

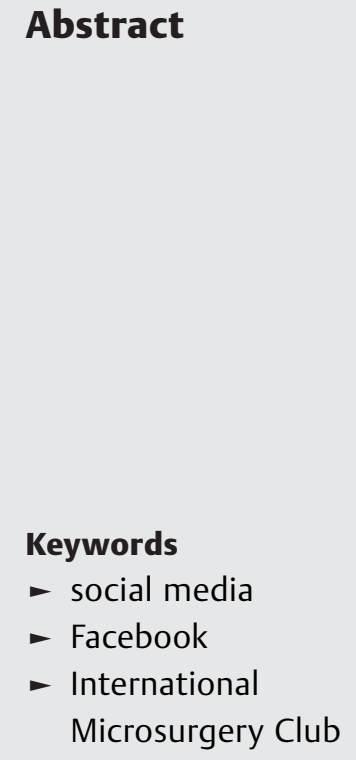

Background This study aimed to determine if International Microsurgery Club (IMC) is an effective online resource for microsurgeons worldwide, in providing an avenue for timely group discussions and advice regarding complicated cases, and an avenue for collaboration and information sharing.

Methods All posts on the IMC Facebook group from member 1 to 8,000 were analyzed according to inclusion criteria and categorized into three categories-case discussion, question, and information sharing. Posts were retrospectively analyzed for number of responses, time of responses, number of "likes," number of treatment options, time of day, and demographics of authors and responders.

Results A retrospective analysis of 531 cases showed an average response rate of $75.7 \%$ within 1 hour and as membership grew. The response rate stabilized averaging between 72.5 and $78 \%$ across all times of the day. An average of 11.8 microsurgeons was involved per case discussion, and 5.7 treatment options were provided per case. Conclusion IMC is shown to be an effective resource to allow microsurgeons to access timely advice from other microsurgeons without time and distance limitation, and to have interactive group discussions on complicated cases.
The role of online learning has become invaluable in today's modern age of technology. The availability of online resources, including journals, websites, YouTube, and social networking websites, provides instant access to answers to clinical questions. Microsurgery is no exception in the developing network of online learning. ${ }^{1-4}$ Mabvuure et al reported in 2014 that $82 \%$ of plastic surgeons from the United Kingdom participated in at least one online platform. ${ }^{5,6}$ Since Facebook started in 2006, it has become the largest social media platform in the world. In 2019, Facebook is the third most visited website in the world with more than 2 billion users, followed by Google and YouTube., ${ }^{1,6-9}$ received

April 27, 2019

accepted after revision

January 4, 2020

published online

February 28, 2020
Copyright $\odot 2020$ by Thieme Medical

Publishers, Inc., 333 Seventh Avenue, New York, NY 10001, USA. Tel: +1(212) 760-0888.
DOI https://doi.org/ 10.1055/s-0040-1702158. ISSN 0743-684X. 
According to a study conducted by Clinical Care Options in 2017 , more than $50 \%$ of physicians in the United States access social media for professional purposes. ${ }^{10}$ Among those physicians, $46 \%$ reported they also used Facebook for personal use. ${ }^{10,11}$ For these reasons, Facebook was chosen for the development of the International Microsurgery Club (IMC). ${ }^{1,6,7,12,13}$ Medical interest groups are not new to Facebook. There exist hundreds of medical groups already; however, their utility is often limited by low number of members or inactivity. ${ }^{14}$ Some examples of effective medical interest groups include the Interactive Plastic Surgery Network (IPSN), Orthobullets, and Handsurgery.cn. IPSN is an established plastic surgery group on Facebook, started in 2012, who provide case discussion in all fields of plastic surgery. ${ }^{7,15,16}$ Orthobullets.com and Handsurgery.cn are customized websites which provide platforms to share information and create discussion. ${ }^{17,18}$ As microsurgery is a highly specialized field, surgeons with this specific knowledge, experience, and skills are a rare commodity. The aim of IMC is to provide connection and access to global microsurgeons for online consultation, discussion, and collaboration in an easy and economical way that previous conventional methods of connection, such a journals, textbooks, and conferences, fail to provide.

IMC is a professionals-only semiclosed group on Facebook, initiated by Dr. Tommy Nai-Jen Chang in May 2016. Membership is granted on validation of title, department, hospital, and speciality. ${ }^{19,20}$ As of August 2019, more than 11,600 members have joined the group and now IMC is one of the largest online microsurgery education groups worldwide. ${ }^{2,19}$ The aim of IMC is to provide an online forum for microsurgeons worldwide to communicate effectively. 2,4 Members are able to post cases, questions, and clinical photos/videos in a format which most people are familiar with, due to the popularity of Facebook. ${ }^{2-4,21}$ The posts not only provide valuable advice to surgeons but also provides opportunities for collaborations in research and learning resources for surgical trainees. Access to this network of information on IMC is free. $2,22-24$

Only posts related to microsurgery are authorized to be posted on IMC, with an exception for information regarding conferences. Information on conferences may be relevant for the members of IMC, as the membership consists of predominantly plastic surgeons and orthopaedic surgeons. With the growth and activity seen in IMC, we hypothesize that IMC will continue to grow and provide a valuable resource of online learning and consultation for microsurgeons worldwide.

\section{Methods}

All posts from the date of establishment (May 6, 2016) until February 17, 2018, 8,000 members were retrospectively analyzed and categorized into three broad categories-case discussion, questions, and information sharing. Groups of 1,000 members were allocated a group number sequentially. Inclusion criteria for case discussion posts included posts which provided a clear history, photos/videos, and other important investigations such as X-rays. Inclusion criteria for information sharing posts included conference information and medical news.

Case discussion posts were analyzed for number of responses, timing of responses, treatment options provided, number of members involved in discussion, time of day, demographics of members, and number of "likes" (which includes other positive icons such as happy, surprised, and love after Facebook software updated). Question and information sharing posts were analyzed for number of "likes" and number of responses. Pearson's chi-square test was used for the analysis of time of day and subspecialties involved in discussion. Sociograph.io software was used for analysis of post authorship. ${ }^{25}$

\section{Results}

A total of 1,839 posts were analyzed: 944 case-related discussion posts (pre- and postoperation discussions), 740 information sharing posts, and 155 question posts. - Fig. 1A shows a positive correlation between number of members and response rate. As the number of members increases, the response rate also increases. At 8,000 members, $81.4 \%$ of posts received a response within 1 hour, and $74.4 \%$ received at least three responses within 2 hours. - Fig. 1B, C compares all three types of posts. Case-related discussion posts received the most "likes" (includes other positive icons) and "responses," whereas information sharing posts received the least.

A total of 531 clinical cases were included in the study. The average number of cases posted perday was 0.8 . The percentage of responses received within 1 hour was $75.7 \pm 7.1 \%$. The percentage of first responses within 1 hour increased as member numbers increased and then gradually stabilized over time ( - Fig. 1A). An average of $11.8 \pm 8.0$ microsurgeons was involved, and $5.7 \pm 3.8$ options for reconstruction were provided per individual case. The average number of "likes" per case was 46.3 (- Fig. 1B, C).

It was observed that the number of responses was correlated with certain case topics and subspecialties in microsurgery. For example, we compared hand surgery cases with peripheral nerve surgery cases. Hand surgery is thought to be one of the most popular fields, and peripheral nerve surgery to be less popular. The number of responses and response rate within 1 hour for cases involving peripheral nerve surgery were found to be lower than that for cases involving hand surgery. However, this result was found not to be statistically significant (-Table $\mathbf{1}$ ).

In order to observe the effect of time zones on response time, we divided 24 hours into four equal time intervals based on Taiwan time (GMT +8$)$. It was found that across all time intervals, the percentage of posts with a first response time within 1 hour ranged between 72.5 and $78 \%$. This was found not to be statistically significant $(p=0.739)$ (-Fig. 2A).

An increase in diversity of members of IMC also expands the breadth of knowledge and experience held within the network. Facebook background analysis of members' 


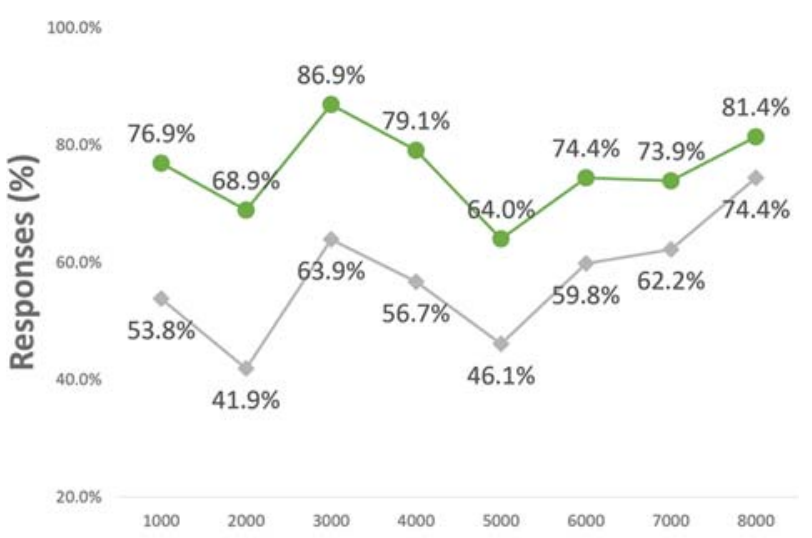

A Member number

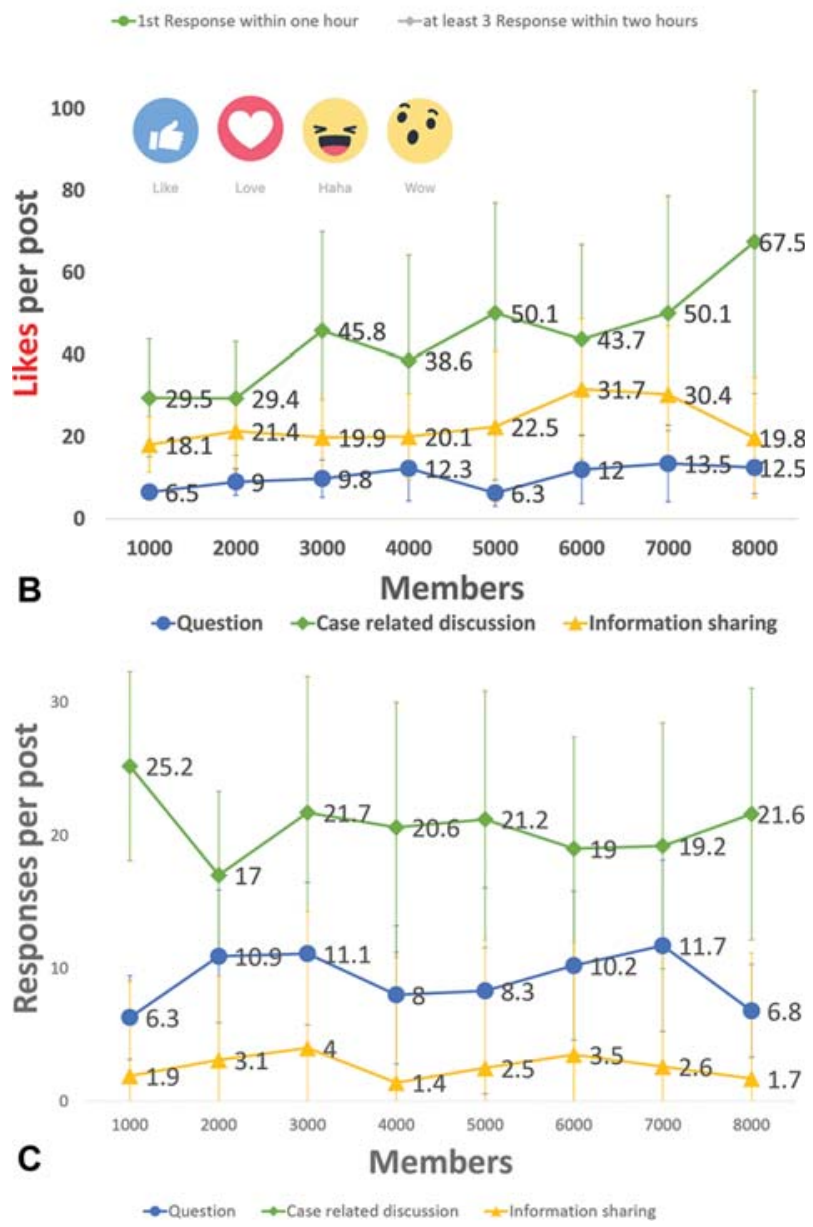

Fig. 1 (A) The response rate (\%) of "first response within 1 hour" and "at least three responses within 2 hours after the initial post." (B) The number of "likes and other positive emoticons" according to post type (case-related discussion, question, information sharing) showed that the case-related discussion attracted the most discussion and interaction over time. (C) The number of responses per post for each type of post category (case-related discussion, question, and information sharing).

country of practice showed the top five countries are India, Egypt, Pakistan, Taiwan, and the United States. Sociograph software reports show an upward trend in the number of authors contributing to discussion as membership grows. An average of 72.3 different authors per 1,000 members group was active in posting cases-related discussions, sharing experiences, and information (-Fig. 2B).
Table 1 A comparison of various data points between hand surgery posts and peripheral nerve surgery posts

\begin{tabular}{|l|l|l|l|}
\hline \multirow{2}{*}{} & \multicolumn{2}{|l|}{$\begin{array}{l}\text { Category of } \\
\text { microsurgery }\end{array}$} & \multirow{2}{*}{-Value } \\
\cline { 2 - 3 } & Hand & Nerve & \\
\hline First response within 1 h (\%) & 70.9 & 60.0 & 0.289 \\
\hline 3+ responses within 2 h (\%) & 58.2 & 35.0 & \\
\hline Options of reconstruction & $6.3 \pm 4.3$ & $3.6 \pm 2.0$ & \\
\hline $\begin{array}{l}\text { Members involved in } \\
\text { discussion }\end{array}$ & $11.9 \pm 8.3$ & $6.1 \pm 3.9$ & \\
\hline Case number & 134 & 20 & \\
\hline
\end{tabular}
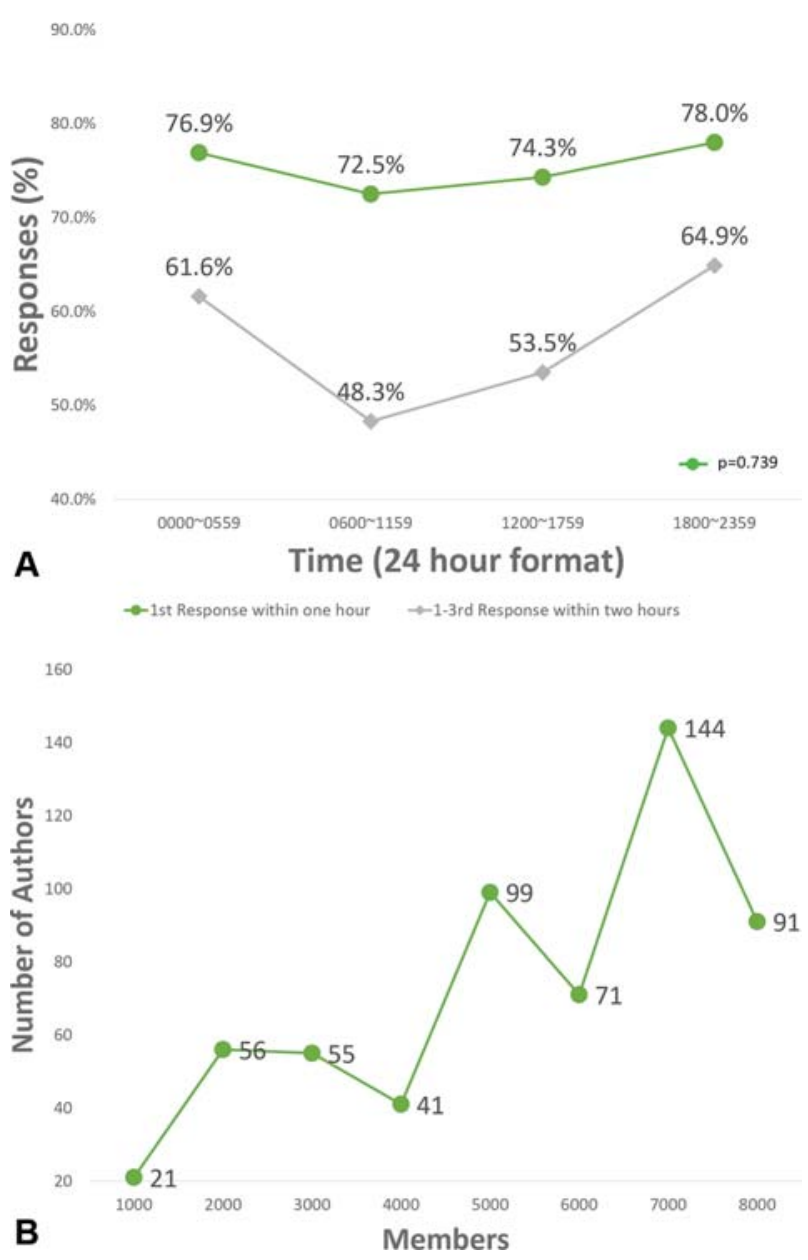

Fig. 2 (A) The response rate at different time intervals of the day (based on Taiwan GST +8). (B) Number of different authors involved in discussion increase as member numbers also increase.

\section{Case Presentation}

Case 1: Posted by Dr. Samir Ghoraba (Egypt)

A 46-year-old woman presented with a wrist amputation and replantation. Despite brisk capillary refill postoperatively, the performing surgeon, Dr. Ghoraba, was concerned about venous insufficiency to the replanted hand. He posted on IMC requesting member input. The first response was received 11 minutes after the post, 11 responses received within 1 hour, and 17 members worldwide were involved in 
the case discussion. Seven different treatment suggestions were offered and discussed by members, including (1) observation, (2) re-explore and perform additional vein anastomoses, (3) shorten the bone $1.5 \mathrm{~cm}$, suture the main arteries and nerves, and find additional veins for anastomosis, (4) release of sutures and evaluate, (5) proximal fasciotomy, (6) use of leeches, heparin, and continuous infusion of dextran, and (7) removal of nail and application of topical nitroglycerin patches near the anastomosis (-Fig. 3A-C).

Based on the advice received, Dr. Ghoraba performed the following: (1) release of all sutures, (2) re-explored the veins and confirmed their patency, (3) performed an additional venous anastomosis, (4) exposed nail beds and applied heparin, and (5) released the fascia between the metacarpals. As a result, the color of the replanted palm appeared pink with good capillary refill immediately postoperatively, and the hand replantation was salvaged successfully (-Fig. 3D). The 13-month follow-up photos showed acceptable functional and aesthetic results after tenolysis and covered with thoracodorsal artery perforator flap ( - Fig. 3E, F).

\section{Case 2: Posted by Dr. Tommy Nai-Jen Chang (Taiwan)}

A 30-year-old woman required resection of an anterior abdominal wall desmoid tumor, measuring $10 \times 10 \times 8 \mathrm{~cm}$ in
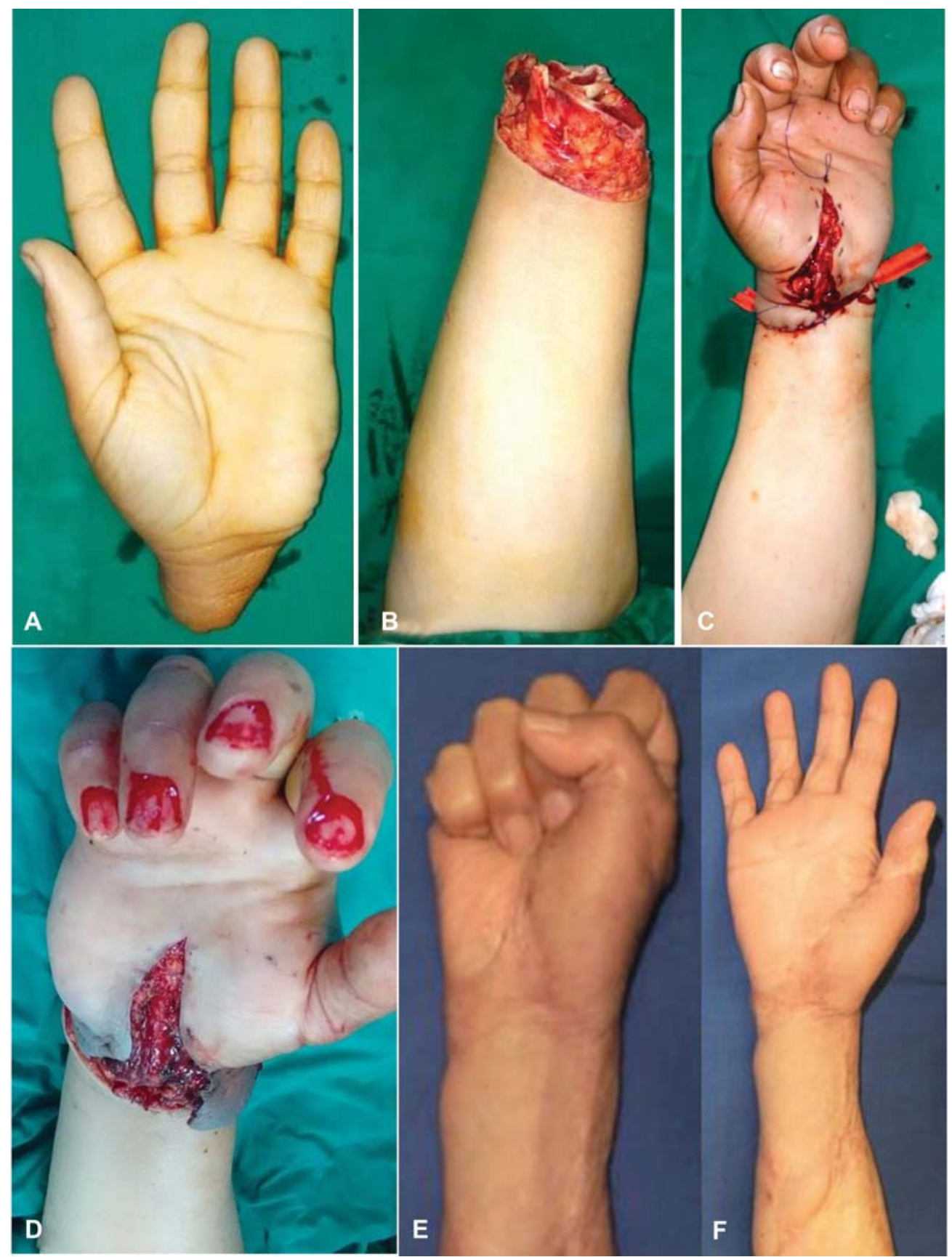

Fig. 3 (A, B) A 46-year-old woman suffered from wrist amputation and received replantation. (C) Postreplantation. Postoperative complication of poor capillary refill after surgery. (D) Post re-exploration. Nails removed with heparin gauze rinse, additional venous anastomosis performed, removal of stitches, and fasciotomy performed. Hand capillary refill restored and became pink. (E, left, and F, right): The 13-month follow-up photos showed acceptable functional and aesthetic results after tenolysis and covered with thoracodorsal artery perforator flap. 
size. Reconstruction was planned around the patient's desire to bare children in the future. Due to the complexity of the case, Dr. Chang posted on IMC 8 hours prior to the operation requesting further reconstruction options. The first response was received 11 minutes after the initial post. Nine responses were received prior to the operation, with involvement of 16 members in discussion. Seven different suggestions were offered and discussed, including (1) reconstruction using a latissimus dorsi myocutaneous flap, (2) reconstruction using a rectus femoris (RF) flap harvested from the anterolateral thigh (ALT) flap, (3) reconstruction using a tensor fascia lata (TFL) flap, (4) use mesh for hernia prevention and reconstruction using a pedicled innervated vastus lateralis and anterolateral thigh flap, (5) use of radiotherapy to reduce the tumor size and reconstruction using a greater omentum flap, (6) reconstruction using a free gracilis flap, (7) use mesh initially and then reconstruct the lateral abdominal muscles with preoperative botulinum toxin, followed by separation of the posterior components (bilateral transversus abdominis release) to achieve direct myofascial closure and reinforcement with a sublayer of mesh in the retrorectus transversus abdominis/ transversalis plane (-Fig. 4A, B).

After the tumor was excised, an abdominal defect to the rectus abdominal muscle, abdominal fascia, and overlying skin remained. After consideration of all suggestions from IMC, Dr. Chang decided to perform a pedicle RF muscle to replace the rectus abdominis (RA) muscle defect, to vascularize the fascia from the TFL, to replace the abdominal fascia with the lateral thigh, and to perform an ALT flap to cover the soft tissue and skin defect. Flap vascularity was monitored. The lateral circumflex femoral artery was used to produce a pedicle chimeric (muscle-fascia-skin) flap. The RF muscle was designed as a functional pedicle muscle, and the motor nerve of the RA was translocated to the T11 intercostal nerve via a $15-\mathrm{cm}$ nerve graft. During pregnancy, respiratory control is used to control muscle movement, rather than knee extension (-Fig. 4C-G).

The operation had no complications and the patient was discharged 10 days postsurgery. Seven months postoperatively, the patient returned to normal activities without complications. She is able to perform abdominal exercises without difficulty and knee extension was normal. On analysis, this case discussion received 187 "likes" and 63 responses.

\section{Discussion}

As technology and infrastructure continue to improve, accessibility to the internet continues to allow the impact

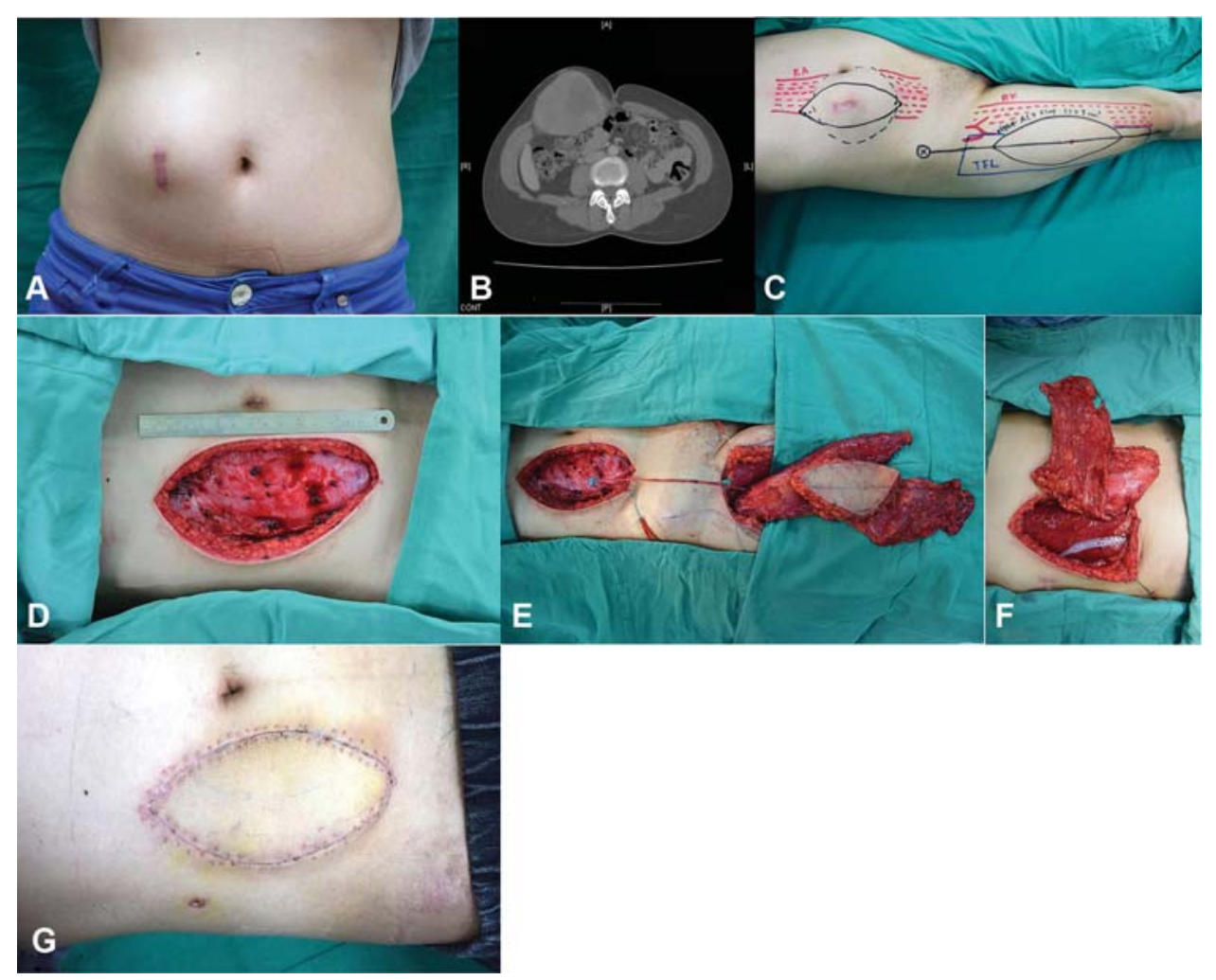

Fig. 4 (A, B) A 30-year-old woman suffered from an anterior abdominal wall desmoid tumor about $10 \times 10 \times 8 \mathrm{~cm}$ in size. (C) The preoperative design. (1) Rectus femoris (RF) to replace the rectus abdominis (RA) muscle as the functional muscle. (2) Vascularized fascia from tensor fascia lata (TFL) and lateral thigh to replace the abdominal fascia. (3). The anterolateral thigh (ALT) flap to fill the soft tissue and skin defect. Everything based on the lateral circumflex femoral artery as a pedicle chimeric flap. (D) The defect after tumor resection, the peritoneum was shown. (E) After pedicle chimeric flap harvest base on lateral circumflex femoral artery. The motor nerve of the rectus femoris was cut and transferred to the T11 intercostal nerve via a 15-cm nerve graft as the pedicle functional muscle. The nerve transfer allows the patient to use the respiration to control the muscle, not the knee extension. (F) After the muscle inset, the proximal sutured to the residual rectus abdominis muscle, and the distal suture to the local fascia close to the inguinal ligament. (G) Sixteen days postoperatively, the wound healed and the donor-site morbidity was subclinical. 
of social media to expand. ${ }^{1,6,13}$ Social media allows access to a greater audience and the ability to communicate information to that audience more effectively. ${ }^{6,26}$ It is common practice for professionals to perform web searches or view instructional videos on "YouTube" rather than shifting through large volumes of textbooks or journal articles. Although instantaneous, the interactive element is lacking. It has become an increasing trend for professional websites to have social media platforms, such as Facebook, Twitter, and LinkedIn, to be integrated within their websites. ${ }^{1,2,4,6,7,9,12,13}$ Compared with previous conventional learning resources, such as conferences, journals, and textbooks, online learning has revolutionized the way knowledge and information is delivered. Previously, by the time textbooks and hardcopy journals were published, much of the information became outdated. Online, any individual with access to the Internet is able to share their own experiences and knowledge to others in a matter of seconds. ${ }^{1-3}$ Social media platforms are reliant on members to build, enrich, nurture, and maintain the content and activity.

The experience and knowledge of senior surgeons worldwide are a treasured commodity. There are several barriers in communicating those experiences and knowledge to others. These include, language, platform barriers, cost, complexities in publishing formats and requirements, and time limitations. ${ }^{2,4}$ Therefore, an online platform, as a costfree resource, may reduce barriers for global surgeons to interact, share experiences, and collaborate with other microsurgeons worldwide. ${ }^{2}$ Microsurgeons can use IMC to learn novel skills and knowledge, form professional friendships, collaborate in research, and hold international conferences. $^{27}$

In this study, we retrospectively analyzed 1,839 posts, including 531 cases, to assess the efficacy of IMC as a reliable and effective online collaboration and consultation system. It has been observed that members post cases on IMC with one of two purposes: (1) to seek recommendations or advice on cases to be performed in the future or to seek advice regarding complications occurring postoperatively, or (2) to share experiences on completed reconstructions. Although both purposes are equally important, in the cases which require advice, it is imperative that the online construct is able to support the need to have timely responses at all times of a 24-hourday. Response rate was found not to be directly proportional to the increase in members. Some possible reasons may be due to (1) sharing of educational information or experiences do not require a response, or (2) treatment options may have been exhausted in early responses. The number of "likes" per discussion is shown to increase with an increase in members. It is observed that even if treatment options were exhausted in early responses, members expressed their support for specific options by "liking" the posted response rather than repeating responses already mentioned. As IMC members increase, the response rate within 1 hour remains stable throughout all time intervals of the day and night. This is particularly relevant for members requiring time critical responses to clinical cases. For example, in case 1 presented earlier1, as the patient had a time critical postoperative complication, urgent advice was needed. Within 1 hour 11 responses with different options were received.

In early statistics performed during the development of IMC (5,000 members mark), we identified a disparity in response rate between different subspecialty fields and topics. The responses in "less popular fields" such as peripheral nerve were statistically significantly slower than the response rate in more popular fields such as hand surgery. As the number of members has increased to 8,000 , the difference in response rates between these two fields is no longer statistically significant. We hypothesize that as member numbers increase, this will provide an increase in the number of diverse specialists who are able to provide timely input into in-depth discussion, even in less popular fields. ${ }^{2,21,28}$

We recognize there are several limitations of IMC. First, privacy is a major concern for any online-based group. In order to maintain confidentiality, our policy requires members to (1) obtain patient consent prior to posting, or (2) deidentify information and photos of patients. Failure to comply with confidentiality will result in nonpublication of posts. Due to varying privacy laws worldwide, the legal responsibility for confidentiality rests upon the author of the post. Privacy and content restriction laws and regulations do not allow content showing exposure of nipples or perineum to be available on Facebook. This regulation limits discussion topic content. ${ }^{23}$ Second, as a semiclosed Facebook group, membership is moderated. Although it is difficult to verify information provided by all members, fake accounts or unrelated people are removed immediately from IMC once identified. Even though the earlier moderation system is not ideal, there has been no major issues within the group in the past 3 years. ${ }^{20,29}$ Third, all attempts are made to moderate advertising from commercial vendors; however, it is also understood that members in IMC may also seek information regarding commercial products. For this reason, IMC has created subgroups to allow commercial vendors to post product advertising. Fourth, photos and videos on Facebook are able to be downloaded by other members of the group. Therefore, copyright and informed consent are mandatory for all posts. Finally, it is important to recognize that members of IMC exist at all levels, from junior doctors to highly experienced microsurgeons; therefore, discretion is required by readers to discern suitability of recommendations made. In order to address the earlier limitations, plans have begun to build a customized website, "International Microsurgery Website", in order to continue to provide greater avenues for connectivity, communication, and consultation in the field of microsurgery. $3,19,30-33$

In a community of more than 11,000 members from around the world, including novice microsurgeons and surgeons from developing countries, IMC holds enormous potential for reach in communication and dissemination of knowledge ( - Tables $\mathbf{2}$ and $\mathbf{3}$ ). 
Table 2 The top 10 countries with IMC members (September 15, 2019)

\begin{tabular}{|l|l|}
\hline Country & Members \\
\hline India & 1,644 \\
\hline Egypt & 1,514 \\
\hline Pakistan & 626 \\
\hline Taiwan & 613 \\
\hline United States & 467 \\
\hline United Kingdom & 337 \\
\hline Bangladesh & 305 \\
\hline Saudi Arabia & 287 \\
\hline Spain & 273 \\
\hline Germany & 250 \\
\hline
\end{tabular}

Table 3 The age/gender distribution of the IMC membership

\begin{tabular}{|l|l|l|}
\hline Age/gender & Male & Female \\
\hline$\leq 24$ & $4.3 \%$ & $1.5 \%$ \\
\hline $25-34$ & $31.9 \%$ & $9.7 \%$ \\
\hline $35-44$ & $29.5 \%$ & $6 \%$ \\
\hline $45-54$ & $9.2 \%$ & $1.3 \%$ \\
\hline $55-64$ & $3.6 \%$ & $0.5 \%$ \\
\hline$\geq 65$ & $1.9 \%$ & $0.4 \%$ \\
\hline
\end{tabular}

Note: There were $41.6 \%$ members between the age of 25 and 34 years and $35.5 \%$ between the age of 35 and 44 years, who were the main learning group (September 15, 2019).

\section{Conclusion}

Through this study, IMC has demonstrated it can be used as an economical, effective, and reliable online consultation system for challenging reconstruction cases. Surgical options are able to be discussed in order to improve patient outcomes. It also provides an effective avenue for communication of professional information such as upcoming conferences and the other related questions. As the number of members continues to grow, it will provide an invaluable resource to microsurgeons, especially to those in isolated regions around the world, or in time critical situations when advice is not readily available. On IMC, microsurgeons are able to advise, educate, and share information with each other at all times of the day equally. We believe that IMC will play a major role in collaboration in global microsurgery in the near future.

\section{Conflict of Interest}

None declared.

\section{References}

1 Branford OA, Kamali P, Rohrich RJ, et al. \#PlasticSurgery. Plast Reconstr Surg 2016;138(06):1354-1365

2 Chang TN. Knowledge revolution through online interactive platform: International Microsurgery Club. Int Microsurg J 2017;1(02):1
3 Messaoudi T, Bodin F, Hidalgo Diaz JJ, et al. Evaluation of a new eLearning platform for distance teaching of microsurgery. Chir Main 2015;34(03):109-112

4 Lin JA-J, Omar UF, Mateev M, Chang TN-J. From virtuality to reality - social media facilitates the interaction between global microsurgeons. Int Microsurg J 2017;1(03):2

5 Mabvuure NT, Rodrigues J, Klimach S, Nduka C. A cross-sectional study of the presence of United Kingdom (UK) plastic surgeons on social media. J Plast Reconstr Aesthet Surg 2014;67(03):362-367

6 McDonald JJ, Bisset C, Coleman MG, Speake D, Brady RR. Contemporary use of social media by consultant colorectal surgeons. Colorectal Dis 2015;17(02):165-171

7 Taglialatela Scafati S, Lapalorcia LM. Social networking and plastic surgery education: running international plastic surgery. Arch Plast Surg 2014;41(04):446

8 Facebook. Facebook [Internet]. Available at: https://www.facebook.com. Cited March 14, 2018

9 Alexa Internet Inc. The top 500 websites on the web [Internet]. Available at: http://www.alexa.com/topsites. Cited March 20, 2018

10 CCO. Social Media Behavior and Attitudes of US Physicians: Implications for Continuing Education Providers [Internet]. 2017. Available at: https://www.clinicaloptions.com/-/media/publications/ 2016/11_2016_social_media_white_paper/white_paper_social_ media.pdf?rev=30f2e6480e044ffd985d50f9ab43e2f5. Accessed February 8, 2020

11 Pew Research Centre. 2015 statistics [Internet]. Available at: http:// www.pewinternet.org/2015/10/08/social-networking-usage2005-2015. Cited March 26, 2016

12 Bender JL, Jimenez-Marroquin MC, Jadad AR. Seeking support on Facebook: a content analysis of breast cancer groups. J Med Internet Res 2011;13(01):e16

13 Wexner SD, Petrucci AM, Brady RR, Ennis-O'Connor M, Fitzgerald JE, Mayol J. Social media in colorectal surgery. Colorectal Dis 2017;19(02):105-114

14 The Treatment Life (TTL) with Dematology \& Plastic Surgery [Internet]. Available at: https://www.facebook.com/groups/healuxttl/? fref $=$ nf. Accessed February 8, 2020

15 Lewis DW. The inevitability of open access. Coll Res Libr 2012; 73:493-506. Accessed February 8, 2020

16 Interactive Plastic Surgery Network [Internet]. Available at: https:// www.facebook.com/groups/international-plasticsurgery/?fref=ts. Accessed February 8, 2020

17 Handsurgery [Internet]. Available at: https://www.handsurgery.cn

18 Orthobullets [Internet]. Available at: https://www.orthobullets. com. Accessed February 8, 2020

19 International Microsurgeons Club [Internet]. Available at: https:// www.facebook.com/groups/1702063276733451. Accessed May 6, 2016

20 Kwon SH, Goh R, Wang ZT, et al. Tips for making a successful online microsurgery educational platform: the experience of International Microsurgery Club. Plast Reconstr Surg 2019;143 (01):221e-233e

21 Pittenger AL. The use of social networking to improve the quality of interprofessional education. Am J Pharm Educ 2013;77(08):174

22 International Microsurgery Journal [Internet]. Available at: https://scitemed.com/Journals/17/International-MicrosurgeryJournal-(IMJ). Accessed February 8, 2020

23 SciTeMed Publishing Group [Internet]. Available at: https://www. scitemed.com/index. Accessed February 8, 2020

24 Facebook: statement of rights and responsibilities [Internet]. 2018. Available at: https://www.facebook.com/legal/terms. Accessed February 8, 2020

25 Sociograph.io. [Internet]. Available at: http://sociograph.io. Accessed February 8, 2020

26 Moorhead SA, Hazlett DE, Harrison L, Carroll JK, Irwin A, Hoving C. A new dimension of health care: systematic review of the uses, benefits, and limitations of social media for health communication. J Med Internet Res 2013;15(04):e85 
27 Lin JA, Hsu AT, Huang JJ, et al. Impact of social media on current medical conferences. J Reconstr Microsurg 2019;35(06):452-461

28 Ryan T, Chester A, Reece J, Xenos S. The uses and abuses of Facebook: a review of Facebook addiction. J Behav Addict 2014; 3(03):133-148

29 Lee GK, Moshrefi S. Discussion: tips for making a successful online microsurgery educational platform: the experience of International Microsurgery Club. Plast Reconstr Surg 2019;143(01): $234 \mathrm{e}-235 \mathrm{e}$

30 Mason KA, Theodorakopoulou E, Pafitanis G, Ghanem AM, Myers SR. Twelve tips for postgraduate or undergraduate medics build- ing a basic microsurgery simulation training course. Med Teach 2016;38(09):872-878

31 International Microsurgery Website [Internet]. Available at: http://imw.global. Accessed February 8, 2020

32 Kwon SH, Lee CH, Hsu AT, et al. Use of social media and an online survey to discuss complex reconstructive surgery: a case of upper lip reconstruction with 402 responses from international microsurgeons. J Reconstr Microsurg 2018;34(06):413-419

33 Kwon SH, Lao WW, Hsu AT, et al. The preferred management of a single-digit distal phalanx amputation. J Reconstr Microsurg 2020;36(4):301-310 